\title{
Spray Characteristics of Elliptical Diesel Nozzles Using Hybrid VOF-to-DPM Transitional Approach in Large Eddy Simulation (LES) Framework
}

\author{
Vivek Kumar*1, Sravan Kumar Nallamothu ${ }^{1}$, Abhijit Patil ${ }^{1}$, Pravin Nakod ${ }^{1}$ \\ ${ }^{1}$ ANSYS Inc, Pune, India \\ *Corresponding author email: Vivek.kumar@ansys.com
}

\begin{abstract}
Elliptical nozzles are known to provide increased spray angle and lower Sauter mean diameter of droplets, thus resulting in improved fuel-air mixing quality that significantly impacts the combustion efficiency and emissions for modern diesel engines. Numerical simulations play an important role in understanding the breakup process in elliptical nozzles and the effect of different parameters such as aspect ratio, injection pressure, back pressure, etc. on the performance of the nozzles. Generally, large eddy simulations (LES) and volume of fluid (VOF) method are combined to understand the characteristics of the elliptical sprays. However, the resolution of the minimum droplet size is limited by the size of the mesh; therefore, such numerical approaches are difficult to adopt for industrial applications. In the current paper, VOF-to-DPM transitional approach and dynamic unstructured mesh refinement are explored to cut down the simulation cost without affecting the simulation accuracy. VOF method captures the core and the larger droplets while Discrete Phase Model (DPM) captures the smaller droplets using the Subgrid approach. VOF-to-DPM transitional approach converts the droplets from VOF framework to DPM framework conserving mass, momentum, and energy. Since smaller droplets are captured in the Subgrid approach, the overall mesh count requirement is considerably smaller than the pure VOF approach. Dynamic unstructured mesh refinement helps to resolve the VOF interface accurately. At the same time, it keeps the mesh count at a reasonable level. Using these approaches, the mechanism of spray breakup and axis switching of elliptical sprays at different operating conditions is studied and the obtained spray tip penetration and Sauter mean diameters are compared with experimental data.
\end{abstract}

\section{Keywords}

Diesel Nozzle, Elliptical, Large Eddy Simulation, Volume of Fluid, Discrete Phase Model, Multiscale Approach, Dynamic Unstructured Mesh Adaption

\section{Introduction}

Liquid jets are commonly employed in various industrial applications such as fuel injection systems in internal combustion engines, gas turbine combustors, spray painting, cleaning, washing, coating, industrial processing, and inkjet printing. The breakup of a liquid jet emanating from the nozzle leads to smaller droplets, thus increasing the interfacial area resulting in higher heat and mass transfer between liquid and the surrounding medium. A high degree of fuel atomization is crucial in obtaining the required fuel-air mixture composition to minimize emissions from diesel engines. While there are several ways to form liquid sprays, the two simplest ways to form liquid sprays are using circular nozzles and elliptical nozzles. Liquid jets produced by elliptical nozzles are known to have higher mixing and reduced breakup length, making them an attractive option for combustor applications [1,2]. Yunyi et al. [3] have shown that the elliptical nozzles produce jets with increased spray angle and lower Sauter mean diameter of droplets compared to circular nozzles, thus resulting in improved fuel-air mixing quality that significantly impacts the combustion efficiency and emissions for modern diesel engines. Lee et al. [4] conducted experiments comparing spray tip penetration 
and spray angle between circular and elliptical nozzles of different aspect ratios. They observed that elliptical nozzles produced jets above a certain aspect ratio, with lower spray tip penetration and higher spray angle than the circular nozzle. They also observed that the fuelair mixture produced by the elliptical nozzle was more homogeneous compared to the circular nozzle. Yu et al. [5] studied the macro spray characteristics of elliptical and circular nozzles under various injection pressures used in a typical diesel engine. They observed that the elliptical nozzles produced higher spray width, lower spray tip penetration, and higher spray angles than the circular nozzle. Through a series of experimental measurements, Hong et al. [6], showed that elliptic nozzles produce jets with larger spray cone angle than circular nozzles. An interesting observation regarding elliptical sprays is that the major and minor axes are switched periodically downstream of the nozzle. The increased instability of the elliptical liquid jets is attributed to this axis-switching phenomenon. Kasyap et al. [7] did several experiments to understand and characterise the axis switching behaviour and breakup length using elliptic nozzles with different aspect ratios. There are several numerical studies focused on understanding the impact of an elliptical nozzle on liquid jet characteristics. Hong et al. [6] carried out experiments to study the spray atomization from a small elliptical nozzle and conducted CFD simulations to investigate the internal nozzle flows. Dolatabadi et al. [8] used volume of fluid (VOF) method to simulate the axis-switching phenomenon observed by Kasyap et al. [7]. Yu et al. [9] used large eddy simulation (LES) model along with the VOF method to simulate the primary atomization of the elliptical liquid jet and compared the spray breakup length, turbulent structures between circular and elliptical jets. Zhang et al. [10] also used a combination of VOF and LES turbulence models to study the elliptical jet breakup with cavitation in the nozzles. All the available CFD works in the literature on elliptic nozzles either entirely focused on what happens inside the nozzle or only on the breakup characteristics.

The present work aims to propose a single simulation framework that can provide detailed insight into the jet breakup process from elliptic nozzles and predict the droplet size distribution. This is achieved using a novel hybrid modeling approach involving coupling of VOF and Lagrangian particle tracking (DPM) with a VOF-to-DPM model transition approach. The advantage of using such an approach is that one can track the movement of the liquid inside and outside the nozzle with a detailed VOF model and switch to the Lagrangian DPM model for tracking the droplets generated by the jet breakup. The transition of liquid mass from multiphase VOF to DPM droplets is taken care of by the VOF-to-DPM model transition mechanism. Dynamic grid refinement is employed, which automatically refines the gas-liquid interface region for accurate resolution of surface instabilities. Several studies focused on developing the Eulerian-Lagrangian hybrid approach and successfully used it to simulate various primary atomization cases such as jet in crossflow, double impinging jet, etc. $[11,12,13,14]$. This hybrid approach is used for the first time to study the spray characteristics for elliptic nozzles. The scope of this paper is to solve the entire flow through an elliptic nozzle using a single simulation workflow and gain as much information about the breakup mechanism as possible. The first part of the CFD study addresses the phenomena of axisswitching associated with elliptic nozzles. We perform detailed CFD simulation using VOF multiphase model with interface tracking schemes. In the second part of the study, we do more simulations on elliptical nozzles under operating conditions employed in a typical diesel engine spray to understand the penetration of the jet for different injection and chamber pressure. The measurements of $\mathrm{Yu}$ et. al [9] are used as a benchmark for evaluating the performance of the CFD models. The third part of the CFD study focuses on predicting the droplet size distribution far downstream of the nozzle, and the results are compared with experimental measurements of Yunyi et al. [3]. The simulations are performed using a two-step workflow. 
The first step involves detailed simulation using VOF-DPM hybrid approach to generate information about the primary breakup and initial condition for the second step which involves Lagrangian tracking of the droplets on a much coarser mesh.

The next section covers the numerical method that talks about the turbulence modeling details, adaptive grid refinement techniques used, solver setting, and a brief description of the VOF-to-DPM hybrid model. We then move to the description of the test cases, results, and discussion section, followed by conclusions.

\section{Numerical Method}

LES with Dynamic Kinetic Energy Subgrid-Scale Model is chosen for modeling turbulence. This combination of models is the most practical way to capture the primary breakup of the elliptical liquid jet. The underlying assumption is the local equilibrium between the transferred energy through the grid-filter scale and the dissipation of kinetic energy at small subgrid scales. The subgrid-scale turbulence is better modeled by accounting for the transport of the subgrid-scale turbulence kinetic energy. The dynamic subgrid-scale kinetic energy model in ANSYS Fluent replicates the model proposed by Kim and Menon [15].

The Volume of Fluid (VOF) method is used to accurately track the shape of the air-water interface. The volume fraction of the phase is explicitly tracked using a geometric reconstruction interface tracking scheme, which can accurately predict the interface curvature and instabilities. Surface tension force is included using the continuum surface force (CSF) model proposed by Brackbill et al. [16]. The tracking of the interface between the liquid and gas is accomplished by the solution of the volume fraction equation for one of the phases. For the liquid phase with no mass transfer involved between liquid and gas, this equation has the following form:

$\frac{\partial}{\partial t}\left(\alpha \rho_{l}\right)+\nabla \cdot\left(\alpha \rho_{l} \vec{v}\right)=0$

For the gas phase, update the volume fraction using the following constraint equation:

$\sum \alpha=1$

To resolve all the relevant liquid structures and to predict the instability and breakup, the VOF model requires fine mesh within the liquid core and at the gas-liquid interface. The hybrid VOFDPM transition mechanism implemented in CFD code ANSYS Fluent searches for all the isolated liquid structures in the domain and these liquid structures are evaluated for their size and sphericity. Near spherical liquid structures are then considered for transitioning into Lagrangian droplets. Jochen et al. [13] have described the finer details of this transition mechanism and how adaptive mesh refinement can optimise mesh and provide a faster simulation turnaround time.

In the DPM model, the fluid phase is treated as a continuum by solving the Navier-Stokes equations in the Eulerian frame, while the dispersed phase droplets are tracked in the Lagrangian frame through the calculated flow field. The dispersed phase exchanges mass, momentum, and energy with the fluid phase. This treatment allows to resolve the large eddy structures at the mesh level and capture the liquid phase and gas phase interaction with accuracy. 
Mesh refinement using Adaptive Mesh Refinement (AMR) near the liquid-gas interface captures the interfacial instability and instability waves. During the breakup process, liquid structures of various shapes and sizes are produced. Spherical drops falling within a specific diameter range transition to discrete phase model droplets. The mesh is coarsened back to its baseline level during the transition process even before the DPM droplets are injected into the computational domain. This approach optimize the overall mesh count and utilises fine mesh only in the primary atomization region. In the following sections, LES and VOF models, along with VOF-to-DPM model transition wherever applicable, are used to understand the axis switching behaviour of elliptical liquid jets and breakup characteristics.

In the current study, simulations for validating three different experimental studies are performed. The same solution settings are used for all three simulations, as shown in Table 1. For pressure-velocity coupling, the Pressure-Implicit with Splitting of Operators (PISO) method is used, which performs two additional corrections for pressure and velocity: neighbour correction and skewness correction. For pressure interpolation, the PREssure STaggering Option (PRESTO!) is used, which uses the discrete continuity balance for a "staggered" control volume about the face to compute the "staggered" face pressure [17].

Table 1: Common solution settings used for simulations

\begin{tabular}{|c|c|}
\hline Pressure-Velocity Coupling & PISO \\
\hline Pressure Discretization & PRESTO! \\
\hline Momentum Discretization & Bounded Central Differencing \\
\hline Turbulence Discretization & Second Order \\
\hline Transient Discretization & First Order Implicit \\
\hline
\end{tabular}

\section{Results and Discussion}

\section{Axis switching behaviour of elliptical sprays}

Kasyap et al. [7] has conducted experiments with one circular and five elliptical nozzles of different aspect ratios at low flow rate conditions and have characterized the axis switching behaviour of elliptic nozzles. In the current study, nozzle E3 with an aspect ratio of 2.97 from Kasyap et al. is used. The dimensions of the E3 nozzle considered for simulation and material properties used, are provided in Table 2.

Table 2 - Description of the nozzle (E3) from Kasyap et. al.

\begin{tabular}{|c|c|}
\hline Major axis $(\mathrm{mm})$ & 4.43 \\
\hline Minor axis $(\mathrm{mm})$ & 1.49 \\
\hline Length $(\mathrm{mm})$ & 100 \\
\hline Aspect ratio & 2.97 \\
\hline
\end{tabular}

A cylindrical entrance section converging into an elliptical channel is considered for simulation. Experiments are performed with water with a density of $995 \mathrm{~kg} / \mathrm{m}^{3}$, viscosity of $0.802 \times 10^{-3}$ Pa.sec, and surface tension of $0.0728 \mathrm{~N} / \mathrm{m}$. A cylindrical domain is considered around the jet representing the ambient air at atmospheric pressure. The geometry and mesh used in the simulation are shown in Figure 1. An initial mesh consisting of 4 million hexahedral elements is generated and refined during the simulation using AMR. 
Figure 1: a) Computational domain used for the axis switching simulation. b) Cross-section showing the initial hexahedral mesh used for the simulation

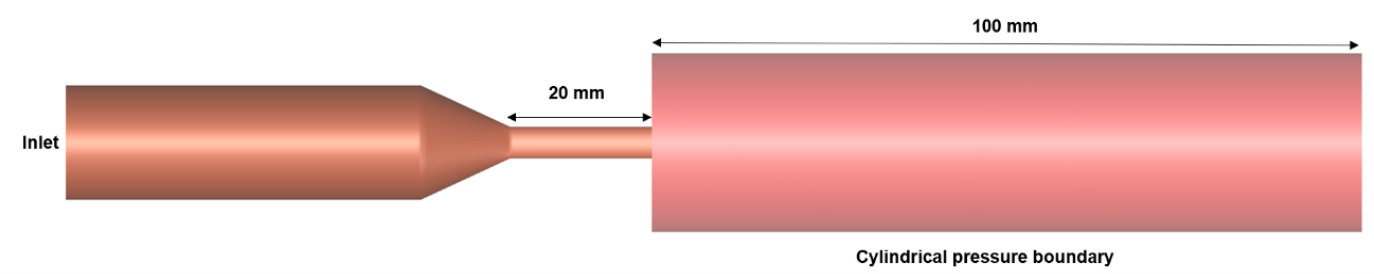

(a)

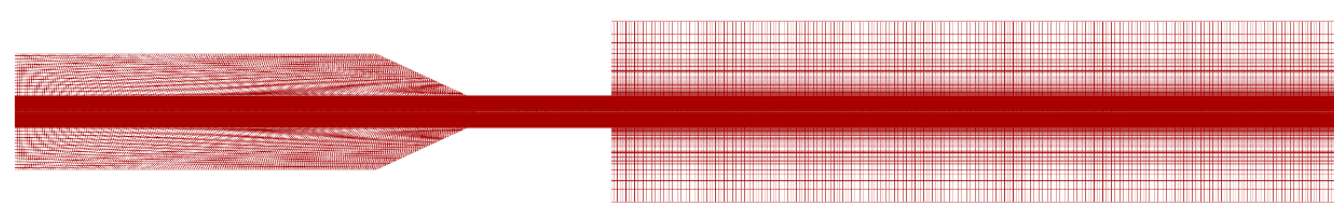

(b)

In this study, VOF multiphase model combined with the LES turbulence model is used to resolve the interfacial instabilities. A VOF-to-DPM transition model is not required since the jet doesn't disintegrate into very small droplets for the chosen conditions. Simulations are run till $1 \mathrm{~ms}$ of physical flow time, and the results are compared with experiments.

Figure 2: Axis switching phenomena in the liquid jet from the E3 elliptic nozzle. (a) and (c) showing experiments in major and minor axis plane, courtesy Kasyap et al. [7], (b), and (d) showing results from simulation
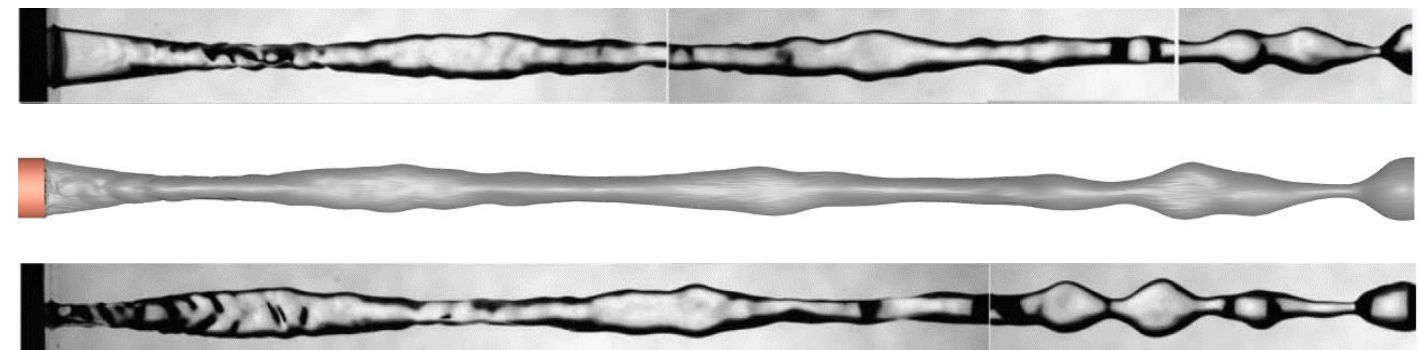

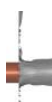

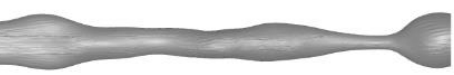

A comparison of the axis switching behaviour obtained from CFD simulation with that of experiments is shown in Figure 2. The simulation results are compared with experimental photographs from Kasyap et al. [7] in both major axis and minor axis views.

Mesh independent study has been done using AMR to check the impact of mesh size on the axis-switching wavelength. Mesh was selectively refined by increasing the levels of adaption. The comparison of axis-switching wavelength for $\sqrt{W e}$ value of 10.58 using different adaption levels is provided in Table 3. Since increasing mesh adaption level has an insignificant impact on the axis switching wavelength, simulations with different weber numbers were performed using one level of mesh adaption. 
Table 3: Comparison of dimensionless axis switching wavelength at different mesh densities

\begin{tabular}{|c|c|c|c|}
\hline S. No & $\begin{array}{c}\text { Number of mesh } \\
\text { elements }\end{array}$ & Adaption level & $\begin{array}{c}\text { Dimensionless Axis } \\
\text { switching } \\
\text { wavelength }\end{array}$ \\
\hline 1 & 5.2 million & 1 & 10.11 \\
\hline 2 & 9.1 million & 2 & 10.74 \\
\hline
\end{tabular}

Three different conditions with different weber number criteria are simulated, and the nondimensional axis switching wavelength is compared with the results obtained by Kasyap et al. in Figure 3. The close resemblance of the simulation result with experimental data validates the accuracy of the models considered in CFD simulation.

Figure 3: Comparison of non-dimensional axis switching wavelength with the square root of weber number

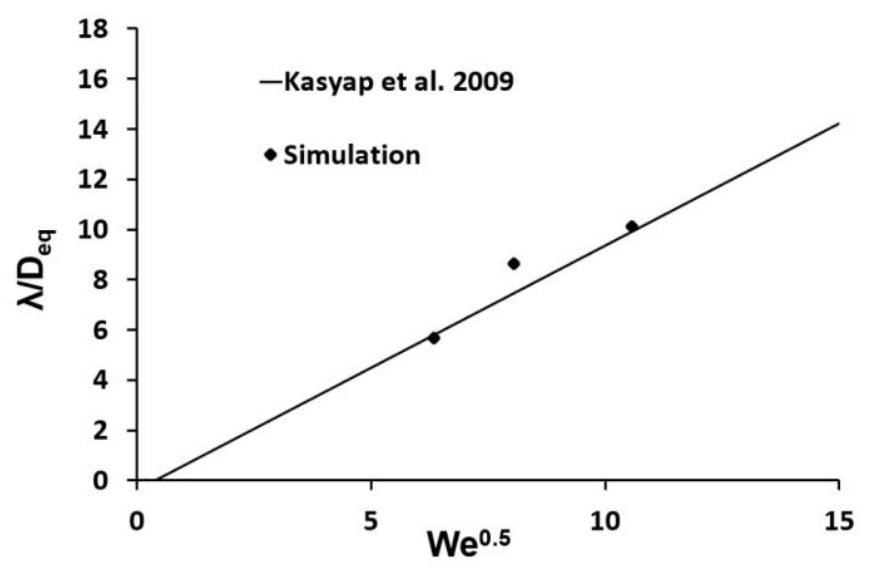

\section{Study of elliptical jet penetration}

Elliptical liquid jets are known to result in lower jet penetration and increased width of the spray. Yu et al. [9] has performed experiments for various elliptic nozzles under typical diesellike conditions. For the present study, one of the nozzles used has been simulated under three different injection conditions.

Table 4: Geometry parameters \& operating conditions

\begin{tabular}{|c|c|c|c|}
\hline \multicolumn{2}{|c|}{ Nozzle geometric parameters } & \multicolumn{2}{c|}{ Process parameters } \\
\hline Major axis $(2 \mathrm{a})[\mu \mathrm{m}]$ & 189.3 & Injection & $50 \mathrm{MPa}$ \\
\hline Minor axis $(2 \mathrm{~b})[\mu \mathrm{m}]$ & 135.2 & Pressure & $90 \mathrm{Mpa}$ \\
\hline Orifice length $[\mathrm{mm}]$ & 1.23 & Back Pressure & $3 \mathrm{Mpa}$ \\
\hline Hydraulic diameter $[\mu \mathrm{m}]$ & 150.9 & Back Temperature & $300 \mathrm{~K}$ \\
\hline
\end{tabular}

Table 4 lists nozzle geometric parameters and the process conditions used in the simulation. Experiments are performed with biodiesel fuel with a density of $876 \mathrm{~kg} / \mathrm{m}^{3}$, a viscosity of $3.96 \times 10^{-3} \mathrm{~Pa} . \mathrm{sec}$, and surface tension of $0.0352 \mathrm{~N} / \mathrm{m}$. Figure 4 shows the cylindrical computational domain with $80 \mathrm{~mm}$ length and $25 \mathrm{~mm}$ radius and initial mesh used to simulate elliptical liquid jet breakup. A body of influence (BOI) is used near the nozzle exit to obtain a refined mesh. 
Figure 4: Computational domain and initial mesh used for simulations
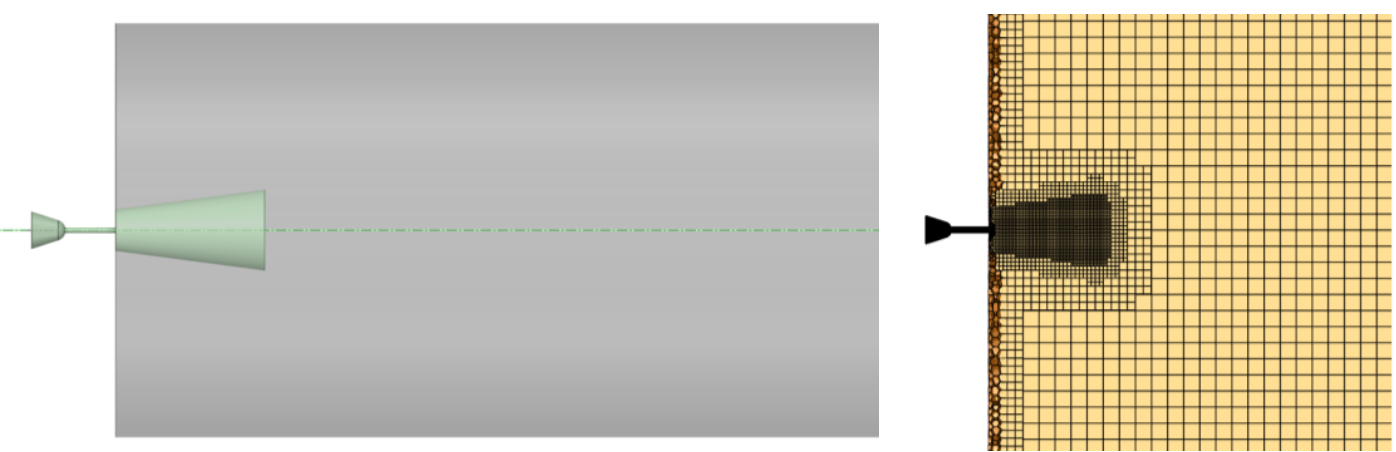

Yu et al. [9] performed mesh independence studies by comparing the inlet pressure obtained in CFD simulation as a function of mesh size in the nozzle. It was found that a mesh size of 2 microns is needed to obtain mesh independent results. Based on this study, a mesh size of 2 microns is used in the nozzle, and a mesh size of 10 microns is used in the sac and the domain outside of the nozzle. Far away from the nozzle, the elements are progressively increased in size to minimize the total element count. All the simulations are performed with a time step size of 10 nanoseconds until 1 milli-second of flow time is reached.

Figure 5: Localized mesh refinement near the liquid jet by dynamic mesh refinement

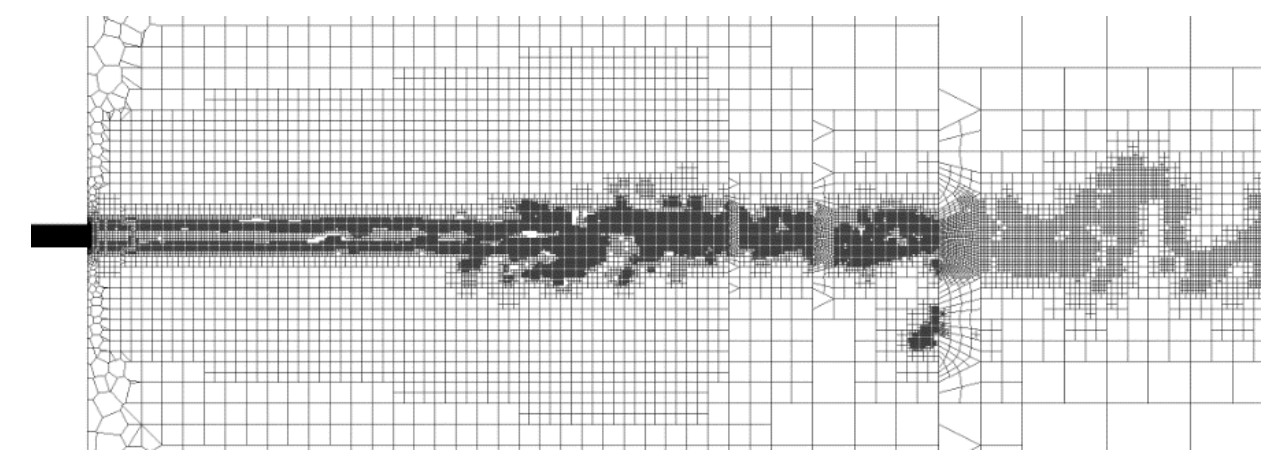

Figure 6: Breakup of a liquid jet showing only larger liquid clusters and their breakup into ligaments and droplets at $90 \mathrm{MPa}$

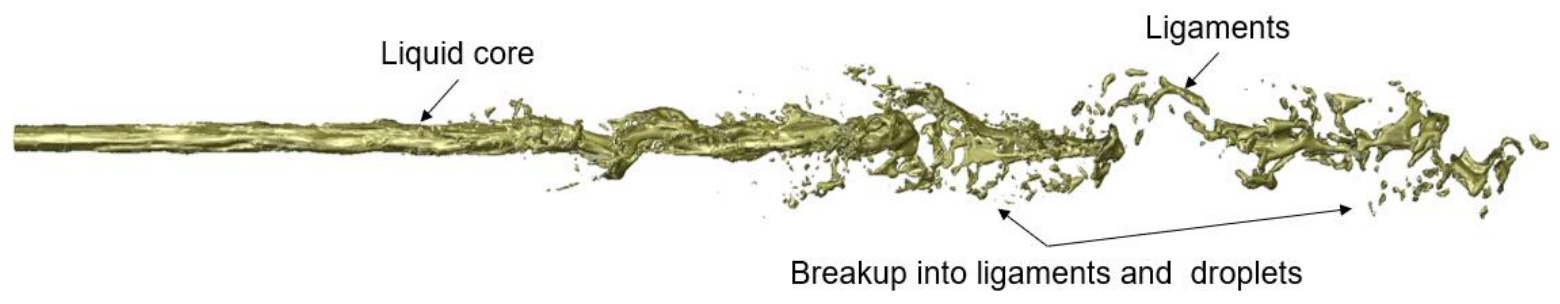

The initial mesh used undergoes dynamic mesh refinement as the gas-liquid interface of the liquid jet evolves with time, as shown in Figure $\mathbf{5}$ for one of the simulations. As the liquid jet undergoes breakup, larger liquid clusters are formed and are broken further into droplets. These droplets resolved with dynamically refined mesh are transitioned into the Lagrangian model using the VOF-to-DPM transition mechanism. Figure 6 illustrates the breakup of liquid jet released from the elliptical nozzle spray at an injection pressure of $90 \mathrm{MPa}$. The breakup of a liquid jet into larger fragments and further break up into ligaments and droplets can be observed. The liquid jet penetration in major and minor axis planes is visualized by plotting the continuous liquid structures resolved by the VOF model, as shown in Figure 7. Figure 8 
(a) shows the complete spray penetration by plotting the liquid structures resolved by the VOF model along with the droplets transitioned into the DPM model. From these images, it is seen that the spray penetration is higher for injection pressure of $90 \mathrm{MPa}$, as expected. Spray tip penetration is compared with experimental data at two different injection pressures and is shown in Figure 8 (b).

As evident from the figure, CFD results are following the experimental trends very well. However, CFD is also over-predicting the spray tip penetration as compared to measurements. This difference could be because of the way spray tip penetration is defined in CFD and measurements. In CFD, it would be challenging to quantify spray-tip penetration by simply looking at the particle tracks as there will always be a few droplets accelerated away or deflected sideways due to gas-phase turbulence. The second reason for the discrepancy could be the mesh resolution chosen for the current analysis. While we followed the meshing guidelines proposed in $\mathrm{Yu}(2018)$ in the primary atomization zone, we chose a very coarse mesh far away from the nozzle where it was expected that the liquid will be present as droplets represented by the DPM model and not as a continuous jet.

Figure 7: Comparison of liquid jet penetration in major and minor axis views between injection pressures of 50 $\mathrm{Mpa}$ and $90 \mathrm{Mpa}$ at three different time instants. Droplets transitioned to the Lagrangian frame are not shown. Nozzle walls are coloured in Brown, and liquid jet is coloured in Cyan

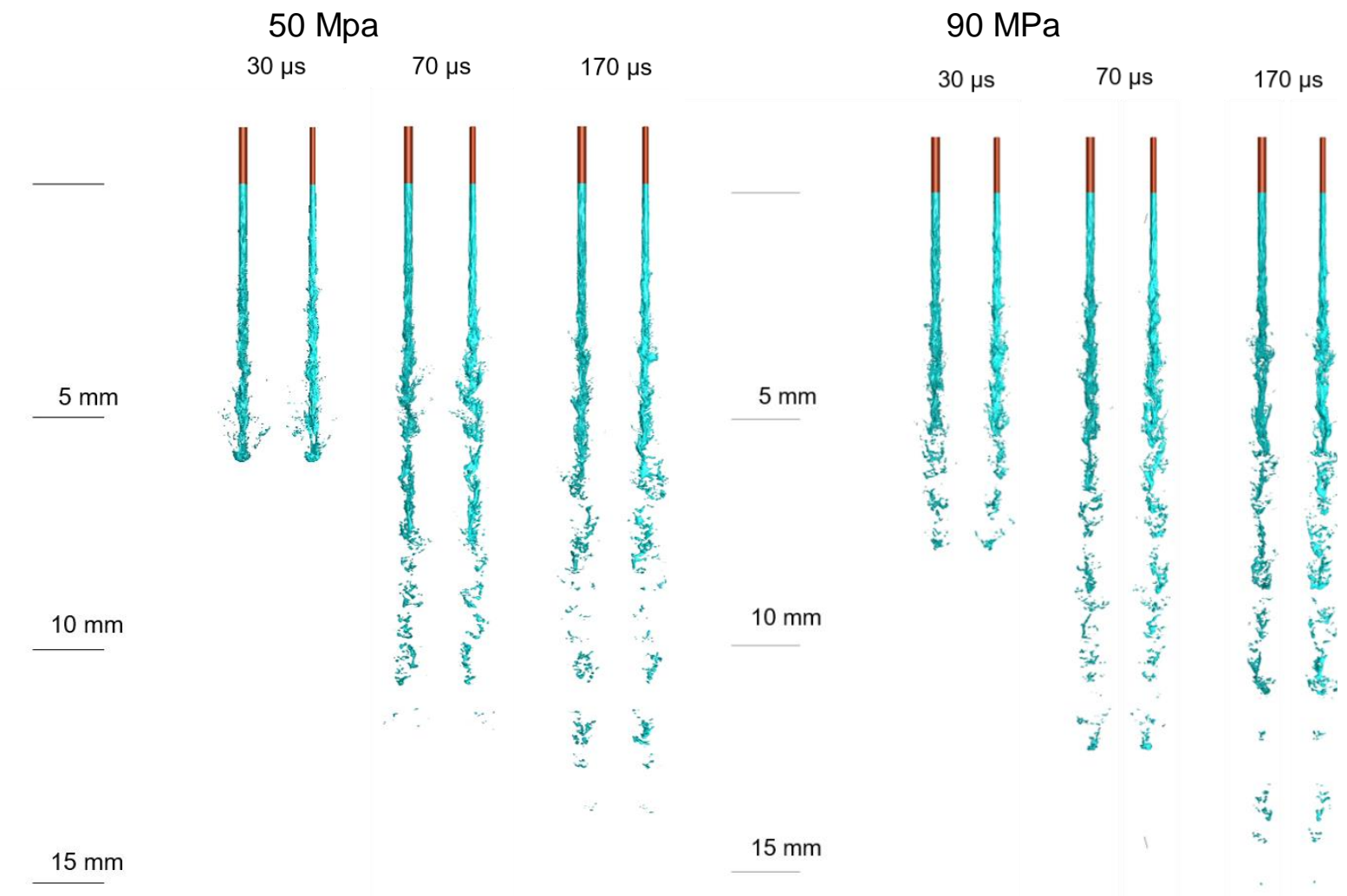

In addition to the visual comparison, spray tip penetration is also measured and compared with experimental data. In the CFD simulation, DPM droplet mass is accumulated starting from the nozzle exit and marching in the axial direction. The axial location where $90 \%$ of the total droplet mass is accumulated is taken as the penetration length. The methodology used to extract the spray penetration. 
Figure 8: (a) Comparison of spray penetration between $50 \mathrm{Mpa}$ and $90 \mathrm{MPa}$ at the time of $170 \mu \mathrm{s}$ in the major axis plane. Liquid jet resolved by the VOF model and droplets transitioned to the Lagrangian model are superimposed for complete spray visualization, (b) Comparison of spray tip penetration with experiments at different injection pressures

(a)

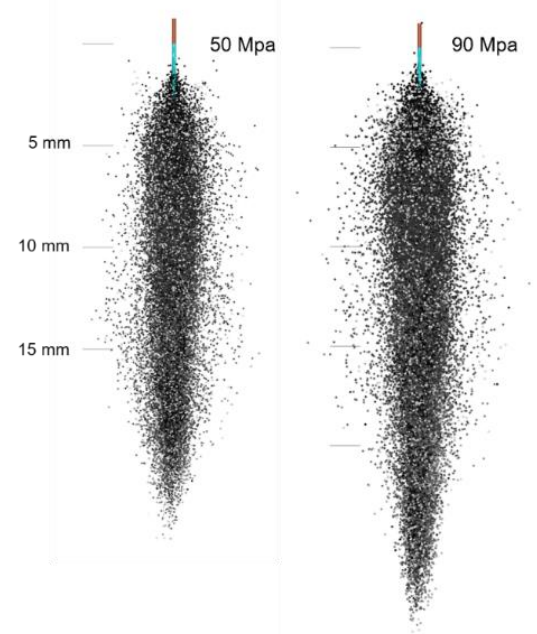

(b)

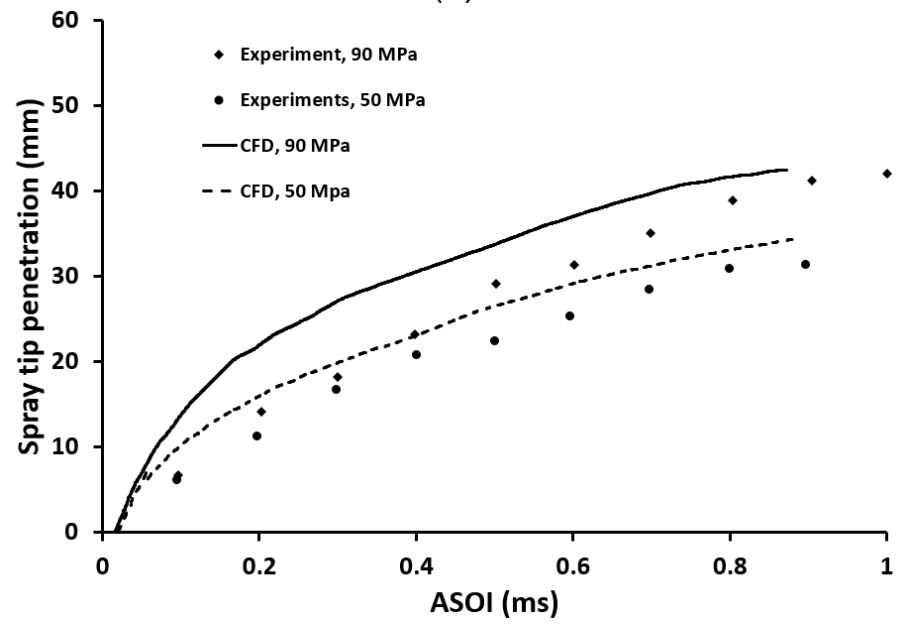

\section{Prediction of Sauter mean diameter of droplets from the elliptical liquid jet breakup}

Yunyi et al. [3] conducted experiments with diesel to study the impact of elliptical nozzles with different aspect ratios on spray characteristics. It was found that the elliptical nozzles produced droplets with smaller Sauter mean diameter (SMD) than the circular nozzle at the same operating conditions. Two elliptical nozzles of different aspect ratios and one circular nozzle are chosen to validate the simulation methodology. The geometry parameters chosen for the numerical study are provided in Table 5. Nozzle numbers and names are kept the same as used in the experimental study for easier identification.

Table 5: Parameters of nozzles considered for simulation

\begin{tabular}{|c|c|c|c|c|}
\hline Nozzle & $\begin{array}{c}\text { Major axis } \\
\text { diameter }(\mathrm{mm})\end{array}$ & $\begin{array}{c}\text { Minor axis } \\
\text { diameter }(\mathrm{mm})\end{array}$ & Aspect ratio & $\begin{array}{c}\text { Hydraulic } \\
\text { diameter, }(\mathrm{mm})\end{array}$ \\
\hline Circular & 0.35 & 0.35 & 1 & 0.35 \\
\hline No. 3 & 0.56 & 0.19 & 2.95 & 0.326 \\
\hline No. 5 & 0.72 & 0.16 & 4.50 & 0.339 \\
\hline
\end{tabular}

The liquid used in the experiments is commercial light diesel fuel with a density of $840 \mathrm{~kg} / \mathrm{m}^{3}$, surface tension of $0.0287 \mathrm{~N} / \mathrm{m}$, and viscosity of 3.25e-3 Pa.sec. Experiments are performed at an injection pressure of $10 \mathrm{Mpa}$ and the droplet SMD was measured using Malvern Particle Sizer. SMD was measured at $200 \mathrm{~mm}$ from the nozzle exit along both major and minor axis radial direction.

The computational domain is chosen similar to the one in the previous study, where a cylindrical domain around the nozzle exit is created that represents ambient conditions. Fully developed elliptical nozzle flow conditions are imposed at the inlet. Mesh sizes are kept similar to the values used for the previous section. In simulations, the methodology used for measuring the droplet diameter at the measuring plane consists of a two-step procedure.

- The hybrid VOF-DPM transition model predicts primary atomization of elliptical liquid jet adjacent to the nozzle till liquid is completely disintegrated and equivalent DPM droplets are released 
- DPM droplets are then tracked in a separate simulation using only the Lagrangian framework until the droplets reach the sampling plane

Figure 9: Sampling planes created to collect droplet SMD data. Sampling planes are coloured orange

This process is also referred to in [13] and reduces the computational expense since the highly resolved VOF-DPM transition simulation is only focused in the region of liquid jet breakup without the need to include lengthy domain needed for sampling. Sampling planes of size 2 $\mathrm{mm} \times 2 \mathrm{~mm}$ are created in the radial direction of both major and minor axis planes for collecting the droplet statistical information. Figure 9 shows the snapshot of droplet spatial distribution along with the planes used for sampling in two different viewing angles. Figure $\mathbf{1 0}$ shows the comparison of SMD across three different nozzles chosen for the simulation. It is seen that the overall SMD predicted by the elliptical nozzles is smaller than the circular nozzle. CFD also predicted a decrease in SMD as the aspect ratio of the elliptical nozzle increased. Experimental data generated by Yunyi et al. showed an increase of SMD with an increase in the radial distance of the spray. Figure 11 shows the comparison of SMD data obtained by simulations with experiments. In CFD simulations, the increase in trend of SMD with radius was observed for circular nozzle. For nozzle-5, and nozzle-3, the SMD did not show a noticeable increasing trend. While SMD values predicted by CFD were well within the range of experimental values, it is observed that the spray width is underpredicted, as can be seen with a lack of data beyond $25 \mathrm{~mm}$ radius for nozzle -3 and $15 \mathrm{~mm}$ radius for nozzle -5 .

Figure 10: Comparison of SMD obtained by simulations across three different nozzles

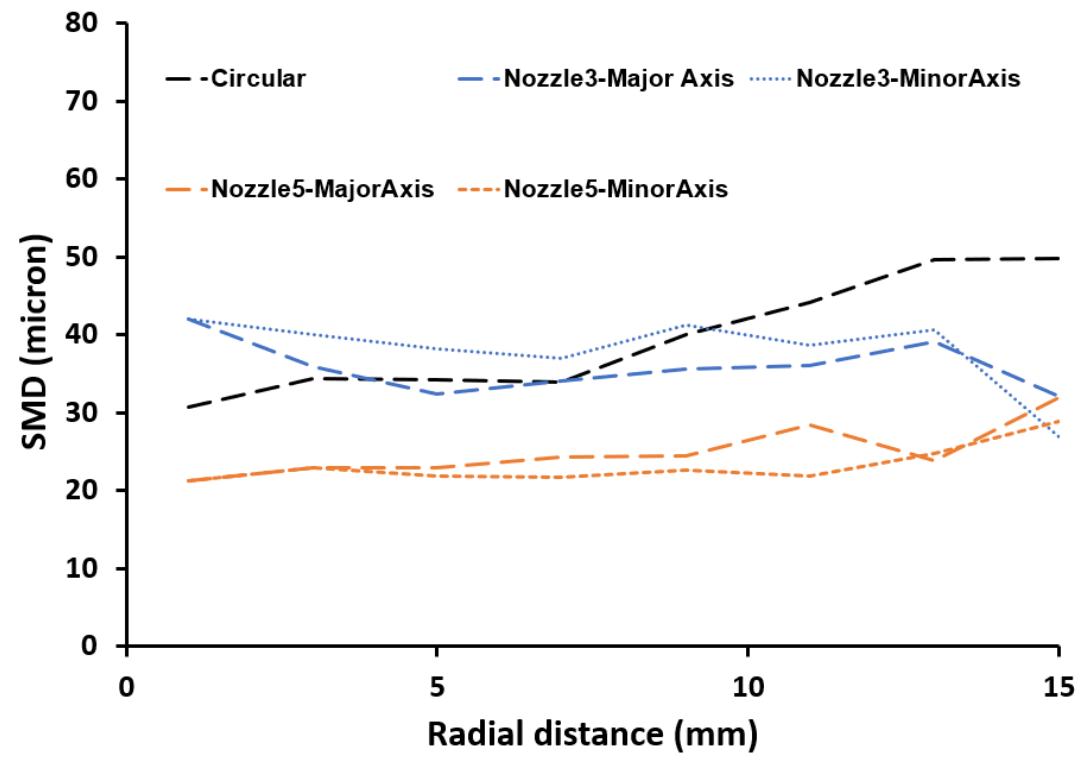


Figure 11: Comparison of SMD with radial distance in major and minor axis direction for (a) Circular nozzle (b) Nozzle-3 and (c) Nozzle-5

(a)
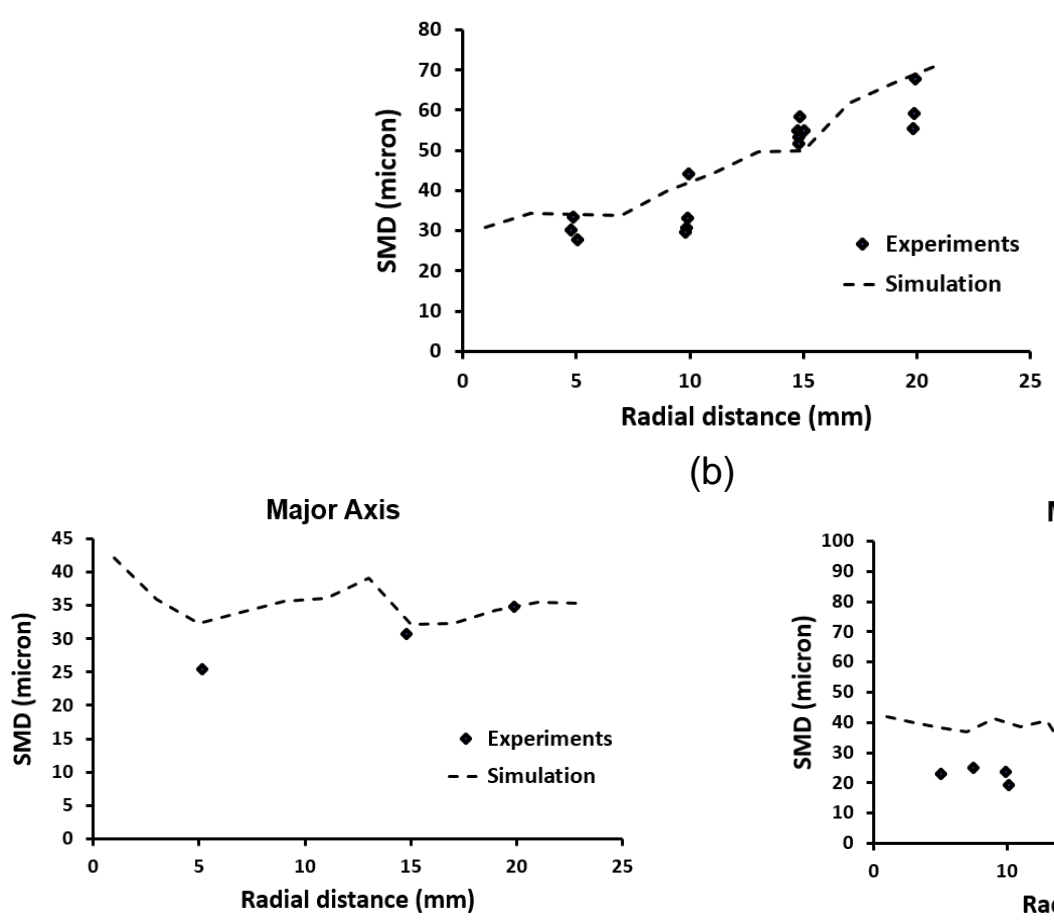

(b)

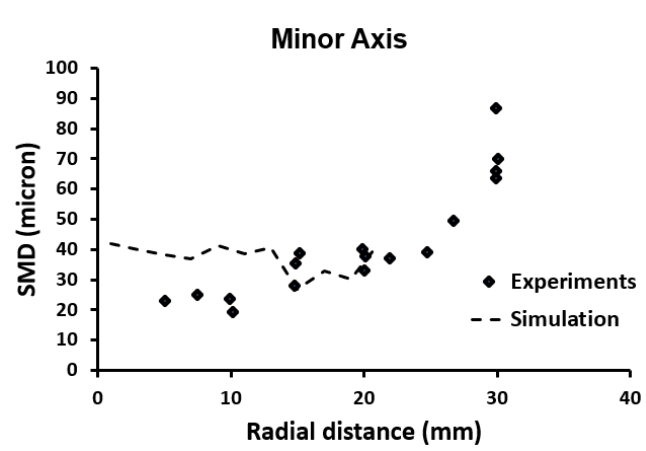

(c)
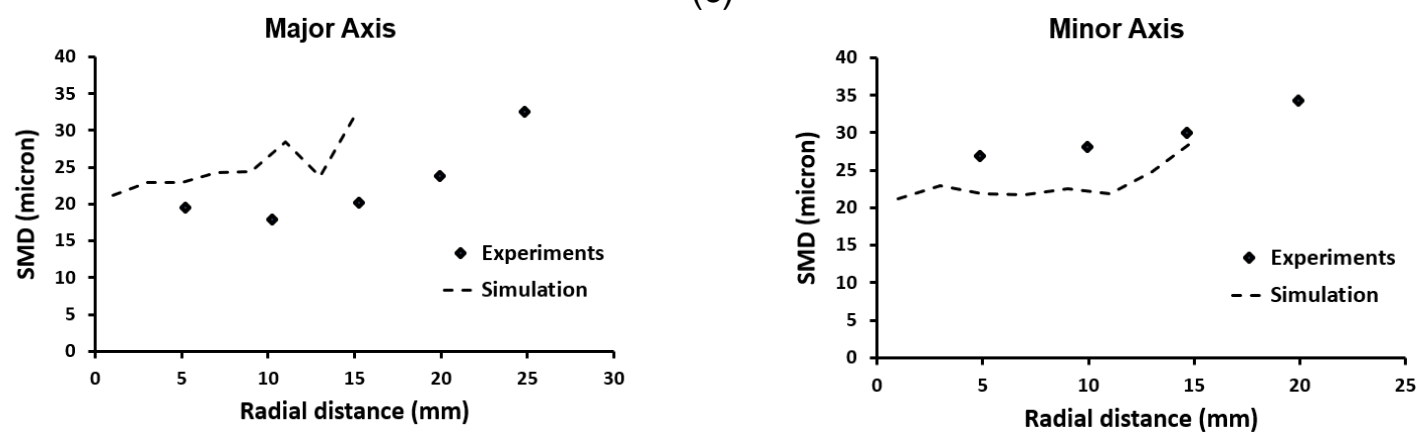

\section{Conclusions}

A hybrid multiscale modeling approach using the LES turbulence model and the VOF multiphase model with the transition to DPM is used on different elliptical jets under different process conditions to validate the approach and understand different aspects of the elliptical jet breakup. At a low flow rate and low weber number, VOF multiphase model shows the axis switching behaviour of elliptical jets. The axis-switching wavelength is compared with measurements showing excellent agreement. The breakup of liquid jets from elliptical nozzles is simulated under diesel-like conditions using the VOF-DPM hybrid approach. The approach is used to validate the CFD results with experimental measurements of $\mathrm{Yu}$. et al at two different injection pressures. In the current study, simulations for circular nozzles are not performed but based on the measurements of $\mathrm{Yu}$ et al it can be deduced that the elliptical nozzles tend to produce quicker atomization and greater jet spread. Finally, a two-step workflow is used to simulate transient tracking of droplets to a distance far from the nozzle orifice. Comparison of Sauter mean diameter of the droplets with experimental measurements of Yunyi et al. gives a match within the range of experimental uncertainty. The approach outlined and used throughout this paper provides a viable and generic approach for characterising industrial 
sprays with reasonable accuracy. This hybrid multiscale approach avoids high computing costs and does not rely on any prior information about the nozzle.

\section{Acknowledgments}

The authors are thankful to Jochen Schuetze, Vinay K. Gupta, Markus Braun, and other members of Ansys development team for their continuous support throughout this activity. Also, the authors are extremely grateful to Shenghao Yu for sharing information about the test nozzles.

\section{Nomenclature}

$\begin{array}{llll}2 a & \text { Major axis } & \text { DPM } & \text { Discrete Phase Model } \\ 2 b & \text { Minor axis } & \text { VOF } & \text { Volume of Fluid } \\ \mu & \text { Viscosity } & \text { LES } & \text { Large Eddy Simulation } \\ \sigma & \text { SurfaceTension Coefficient }[\mathrm{N} / \mathrm{m}] & \text { AMR } & \text { Adaptive Mesh Refinement }\end{array}$

\section{References}

[1] Amini, G., Dolatabadi A., Physics of Fluids (1994-present) 23, 084109 (2011)

[2] Hussain, F., Hussain S. H., J. Fluid. Mech. (1998), vol. 208, pp. 257-320

[3] Yunyi, G., Changwen, L., Yezhou, H., and Zhijun, P., SAE Technical Paper 982546, 1998

[4] Lee, W.C., Kim, I., Koo, W.K., Park, J., and Lee, Y., ICLASS06-203, Proceedings of ICLASS, 2006

[5] Yu, S., Yin, B., Deng, W., Jia, H., Ye, Z., Xu, B., and Xu, H., Fuel, 221 (2018), 28-34

[6] Hong, G.J., Koo, W.K., Lee, W.C., and Chul Na, B., 12th Triennial International Conference on Liquid Atomization and Spray Systems, ICLASS 2012

[7] Kasyap, T.V., Sivakumar D., Raghunandan, B.N, International Journal of Multiphase Flow ,35, 2009, 8-19

[8] Dolatabadi, A., Farvardin, E., Journal of Fluids Engineering, July 2013, Vol. 135, 0713021

[9] Yu, S., Yin, B., Deng, W., Jia, H., Ye, Z., Xu, B., and Xu, H., Atomization and Sprays, 28(8), 2018, 695-712

[10] Zhang, L., Wang, Q., Guan, W., Cao, T., Duan, L., Pachiannan, T., and He, Z., Atomization and Sprays, 29(2), 2019, 177-198

[11] Hermann, M., Journal of Computational Physics, 229,2010, 745-759

[12] Sonawane, R., Kumar, V., and Nakod, P., Proceedings of the Asian Congress on Gas Turbines, GTINDIA2017-4732, V001T04A011

[13] Schütze, J., Gupta, V.K., Aguado, P., Hutcheson, P., Esch, T., and Braun, M., 29th Conference on Liquid Atomization and Spray Systems, ILASS-Europe 2019, 2-4 September, 2019

[14] Kumar, B.A., Kumar, V., Nakod, P., Schütze, J., and Rajan, A., AIAA Scitech 2020 Forum, AIAA 2020-2284, 6-10 January 2020

[15] Kim, W.W., Menon. S., 35th Aerospace Sciences Meeting, Technical Report AIAA-970210, Reno, NVAmerican Institute of Aeronautics and Astronautics. January 1997.

[16] Brackbill, J.U., Kothe, D.B., and Zemach, C., J. Comput. Phys. ,100,1992,335-354

[17] Patankar, S. V., Numerical Heat Transfer and Fluid Flow. Hemisphere, Washington, DC. 1980. 